\title{
Chiral properties of the pseudoscalar meson in two flavors QCD with exact chiral symmetry
}

\author{
Ting-Wai Chiü1,2,3, Tung-Han $\mathrm{Hsieh}^{4}$, and Yao-Yuan Mao ${ }^{1}$ (for the TWQCD \\ Collaboration) \\ ${ }^{1}$ Physics Department, National Taiwan University, Taipei 10617, Taiwan \\ ${ }^{2}$ Center for Quantum Science and Engineering, National Taiwan University, \\ Taipei 10617, Taiwan \\ ${ }^{3}$ Center for Theoretical Sciences, National Taiwan University, Taipei 10617, Taiwan \\ ${ }^{4}$ Research Center for Applied Sciences, Academia Sinica, Taipei 115, Taiwan
}

\begin{abstract}
We perform hybrid Monte Carlo (HMC) simulatons of two flavors QCD with the optimal domainwall fermion (ODWF) on the $16^{3} \times 32$ lattice (with lattice spacing $a \sim 0.1 \mathrm{fm}$ ), for eight sea-quark masses corresponding to pion masses in the range $230-580 \mathrm{MeV}$. We calculate the mass and the decay constant of the pseudoscalar meson, and compare our data with the chiral perturbation theory (ChPT). We find that our data is in good agreement with the sea-quark mass dependence predicted by the next-to-leading order (NLO) ChPT, and provides a determination of the lowenergy constants $\bar{l}_{3}$ and $\bar{l}_{4}$, the pion decay constant, the chiral condensate, and the average up and down quark mass.
\end{abstract}

XXIX International Symposium on Lattice Field Theory

July 10-16 2011

Squaw Valley, Lake Tahoe, California

\footnotetext{
${ }^{*}$ Speaker.
} 


\section{Introduction}

Lattice QCD with exact chiral symmetry [U, ㅁ] is an ideal theoretical framework to study the nonperturbative physics from the first principles of QCD. However, it is rather nontrivial to perform Monte Carlo simulation such that the chiral symmetry is preserved at a high precision and all topological sectors are sampled ergodically.

Since 2009, TWQCD Collaboration has been using a GPU cluster (currently constituting of 250 Nvidia GPUs) to simulate unquenched lattice QCD with the optimal domain-wall fermion (ODWF) [ [3, 团]. Mathematically, ODWF is a theoretical framework which preserves the chiral symmetry optimally with a set of analytical weights, $\left\{\omega_{s}, s=1, \cdots, N_{s}\right\}$, one for each layer in the fifth dimension [3]. Thus the artifacts due to the chiral symmetry breaking with finite $N_{s}$ can be reduced to the minimum, especially in the chiral regime. The 4-dimensional effective Dirac operator of massless ODWF is

$$
D=m_{0}\left[1+\gamma_{5} S_{\text {opt }}\left(H_{w}\right)\right], \quad S_{\text {opt }}\left(H_{w}\right)=\frac{1-\prod_{s=1}^{N_{s}} T_{s}}{1+\prod_{s=1}^{N_{s}} T_{s}}, \quad T_{s}=\frac{1-\omega_{s} H_{w}}{1+\omega_{s} H_{w}},
$$

which is exactly equal to the Zolotarev optimal rational approximation of the overlap Dirac operator. That is, $S_{\text {opt }}\left(H_{w}\right)=H_{w} R_{Z}\left(H_{w}\right)$, where $R_{Z}\left(H_{w}\right)$ is the optimal rational approximation of $\left(H_{w}^{2}\right)^{-1 / 2}$ [1], 目].

Recently we have demonstrated that it is feasible to perform a large-scale unquenched QCD simulation with ODWF, which not only preserves the chiral symmetry to a good precision, but also samples all topological sectors ergodically [0]. Our result of the topological susceptibility agrees with the sea-quark mass dependence predicted by the NLO ChPT [ $[\mathbb{8}]$, and provides the first determination of both the pion decay constant and the chiral condensate simultaneously from the topological susceptibility. Furthermore, our results of the mass and the decay constant of the pseudoscalar meson [Q] also turn out in good agreement with the sea-quark mass dependence predicted by NLO ChPT [ए]] , and from which we obtain the low-energy constants $F, \Sigma, \bar{l}_{3}$ and $\bar{l}_{4}$. With the low-energy constants, we determine the average up and down quark mass $m_{u d}^{\overline{\mathrm{MS}}}(2 \mathrm{GeV})$, and the chiral condensate $\Sigma^{\overline{\mathrm{MS}}}(2 \mathrm{GeV})$.

In this proceeding, we review our results of the mass and decay constant of the pseudoscalar meson presented in Ref. [Q] .

\section{Lattice Setup}

First, we outline our HMC simulation of 2 flavors QCD with ODWF. Starting from the ODWF action $S=\bar{\Psi} \mathscr{D} \Psi$ [B] on the 5D lattice, we separate the even and the odd sites (the so-called evenodd preconditioning) on the 4D lattice, and rewrite $\mathscr{D}$ as

$$
\mathscr{D}\left(m_{q}\right)=S_{1}^{-1}\left(\begin{array}{cc}
1 & 0 \\
M_{5} D_{w}^{\mathrm{OE}} & 1
\end{array}\right)\left(\begin{array}{ll}
1 & 0 \\
0 & C
\end{array}\right)\left(\begin{array}{cc}
1 & M_{5} D_{w}^{\mathrm{EO}} \\
0 & 1
\end{array}\right) S_{2}^{-1},
$$

where $m_{q}$ denotes the bare quark mass, $D_{w}$ denotes the standard Wilson Dirac operator plus a negative parameter $-m_{0}$ (Here $m_{0}=1.3$ in this work.), and $D_{w}^{\mathrm{EO} / \mathrm{OE}}$ denotes the part of $D_{w}$ with 


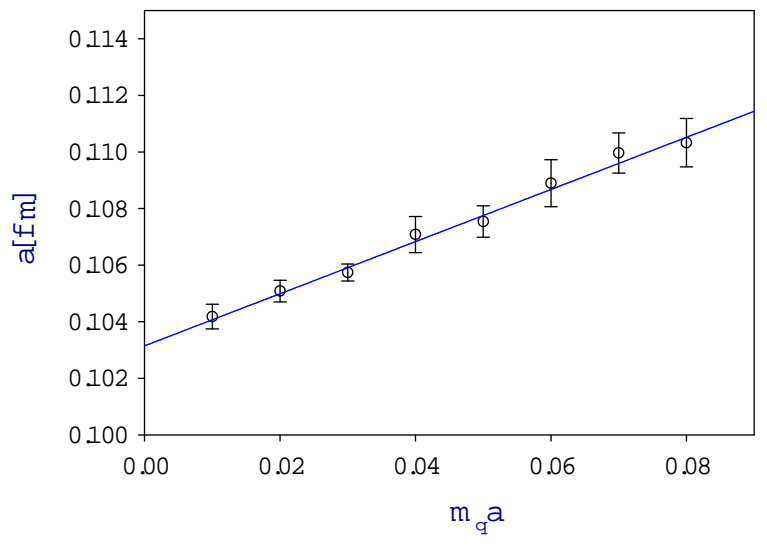

Figure 1: The lattice spacing $a[\mathrm{fm}]$ versus $m_{q} a$ for two flavors QCD with ODWF.

gauge links pointing from odd/even sites to even/odd sites, and

$$
\begin{aligned}
M_{5} & =\left[\left(4-m_{0}\right)+\omega^{-1 / 2}(1-L)(1+L)^{-1} \omega^{-1 / 2}\right]^{-1}, \\
(\omega)_{s s^{\prime}} & =\omega_{s} \delta_{s s^{\prime}}, \quad L=P_{+} L_{+}+P_{-} L_{-}, \quad P_{ \pm}=\left(1 \pm \gamma_{5}\right) / 2, \quad L_{-}=\left(L_{+}\right)^{T}, \\
\left(L_{+}\right)_{s s^{\prime}} & =\left\{\begin{array}{ll}
\delta_{s-1, s^{\prime}}, & 1<s \leq N_{s} \\
-\left(m_{q} / 2 m_{0}\right) \delta_{N_{s}, s^{\prime}}, & s=1
\end{array} ;\right. \\
S_{1} & =M_{5} \omega^{-1 / 2}, \quad S_{2}=(1+L)^{-1} \omega^{-1 / 2}, \quad C=1-M_{5} D_{w}^{\mathrm{OE}} M_{5} D_{w}^{\mathrm{EO}} .
\end{aligned}
$$

Since $\operatorname{det} \mathscr{D}=\operatorname{det} S_{1}^{-1} \cdot \operatorname{det} C \cdot \operatorname{det} S_{2}^{-1}$, and $S_{1}$ and $S_{2}$ do not depend on the gauge field, we can just use $C$ for the HMC simulation. After including the Pauli-Villars fields (with $m_{q}=2 m_{0}$ ), the pseudo-fermion action for 2 flavors QCD $\left(m_{u}=m_{d}\right)$ can be written as

$$
S_{p f}=\phi^{\dagger} C_{P V}^{\dagger}\left(C C^{\dagger}\right)^{-1} C_{P V} \phi, \quad C_{P V} \equiv C\left(2 m_{0}\right) .
$$

In the HMC simulation, we first generate random noise vector $\xi$ with Gaussian distribution, then we obtain $\phi=C_{P V}^{-1} C \xi$ using the conjugate gradient (CG). With fixed $\phi$, the system is evolved under a fictituous Hamiltonian dynamics, the so-called molecular dynamics (MD). In the MD, we use the Omelyan integrator []], and the Sexton-Weingarten multiple-time scale method [ㅁ]]. The most time-consuming part in the MD is to compute the vector $\eta=\left(C C^{\dagger}\right)^{-1} C_{P V} \phi$ with $\mathrm{CG}$, which is required for the evaluation of the fermion force in the equation of motion for the conjugate momentum of the gauge field. Here we take advantage of the remarkable floating-point capability of the Nvidia GPU, and perform the CG with mixed precision [[123]. Furthermore, we introduce an auxillary heavy fermion field with mass $m_{H}\left(m_{q} \ll m_{H}<2 m_{0}\right)$ [प]]. For two flavors QCD, the pseudofermion action (with $C_{H} \equiv C\left(m_{H}\right)$ ) becomes,

$$
S_{p f}^{H}=\phi^{\dagger} C_{H}^{\dagger}\left(C C^{\dagger}\right)^{-1} C_{H} \phi+\phi_{H}^{\dagger} C_{P V}^{\dagger}\left(C_{H} C_{H}^{\dagger}\right)^{-1} C_{P V} \phi_{H}
$$

which gives exactly the same fermion determinant of (D. Devertheless, the presence of the heavy fermion field plays a crucial role in reducing the light fermion force and its fluctuation, thus diminishes the change of the Hamiltonian in the MD trajactory, and enhances the acceptance rate. 


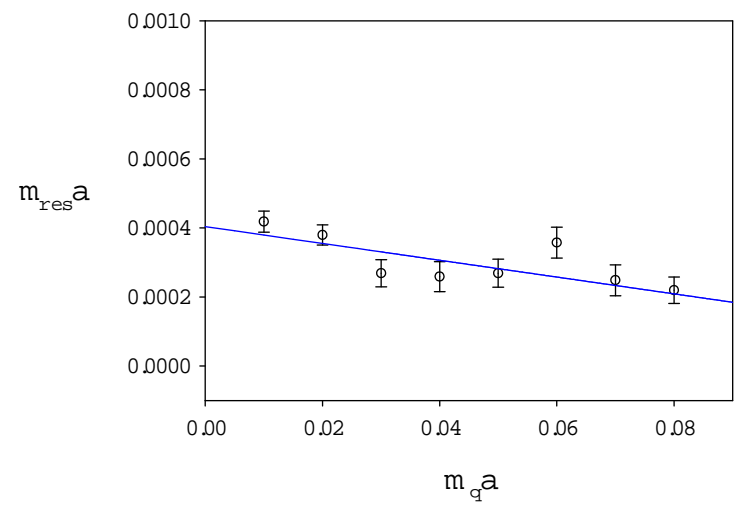

Figure 2: The residual mass versus the sea quark mass for two flavors QCD with ODWF.

Simulations are carried out for two flavors QCD on a $16^{3} \times 32$ lattice, for eight sea-quark masses $m_{q} a=0.01,0.02, \cdots 0.08$ respectively. For the quark part, we use ODWF with $N_{s}=16$, and $\lambda_{\min } / \lambda_{\max }=0.02 / 6.4$. For the gluon part, we use the plaquette action at $\beta=5.95$.

For each sea-quark mass, we perform simulations on 30 GPUs independently, with each GPU generating 400 trajectories. After discarding 300 trajectories for thermalization, each GPU yields 100 trajectories. Thus, with 30 GPUs running independently, we accumulated total 3000 trajectories for each sea-quark mass. From the saturation of the binning error of the plaquette, as well as the evolution of the topological charge, we estimate the autocorrelation time to be around 10 trajectories. Thus we sample one configuration every 10 trajectories, then we have 300 configurations for each sea-quark mass. With a GPU cluster of 250 GPUs, we can simulate 8 sea-quark masses concurrently. It takes about 5 months to complete the simulations for the $\beta=5.95$ ensemble.

We determine the lattice spacing by heavy quark potential with Sommer parameter $r_{0}=$ $0.49 \mathrm{fm}$. The lattice spacing versus the quark mass is plotted in Fig. I. Using the linear fit, we obtain the lattice spacing in the chiral limit, $a=0.1032(2) \mathrm{fm}$, which gives $a^{-1}=1.911(4)(6) \mathrm{GeV}$, where the systematic error is estimated with the uncertainty of $r_{0}$.

For the computation of the valence quark propagator of the 4D effective Dirac operator of ODWF, two methods have been presented in Ref. [15]. In this work, we compute the valence quark propagator with the point source at the origin, and with parameters exactly the same as those of the sea-quarks.

To measure the chiral symmetry breaking due to finite $N_{s}$, we compute the residual mass

$$
m_{r e s}=\left\langle\frac{\sum_{x}\left\langle J_{5}(x) \bar{q}(0) \gamma_{5} q(0)\right\rangle}{\sum_{x}\left\langle\bar{q}(x) \gamma_{5} q(x) \bar{q}(0) \gamma_{5} q(0)\right\rangle}\right\rangle_{\{U\}}=\left\langle\frac{\operatorname{tr}\left(D_{c}+m_{q}\right)_{0,0}^{-1}}{\operatorname{tr}\left[\left(D_{c}^{\dagger}+m_{q}\right)\left(D_{c}+m_{q}\right)\right]_{0,0}^{-1}}\right\rangle_{\{U\}}-m_{q},
$$

where $J_{5}(x)=\bar{\psi}_{x, n} P_{-} \psi_{x, n+1}-\bar{\psi}_{x, n+1} P_{+} \psi_{x, n}$ with $n \equiv N_{s} / 2$, which denotes the chiral density at the central layer in the 5-th dimension, $\left(D_{c}+m_{q}\right)^{-1}$ denotes the valence quark propagator with $m_{q}$ equal to the sea-quark mass, tr denotes the trace running over the color and Dirac indices, and the subscript $\{U\}$ denotes averaging over an ensemble of gauge configurations. It turns out that, after averaging over an ensemble of a few hundreds of independent gauge configurations, $m_{\text {res }}$ seems to be insensitive to the location of the origin $x^{\mu}=(0,0,0,0)$. Thus (2.2) gives a reliable estimate of 
the chiral symmetry breaking due to finite $N_{s}$. In Fig. $\square$, we plot the residual mass versus the sea quark mass. Using the linear fit, we obtain the residual mass in the chiral limit, $m_{\text {res }} a=0.00040(4)$, less than $5 \%$ of the lightest sea quark mass. Thus we confirm that the chiral symmetry is preserved to a good precision in our simulation. In the following, it is understood that each bare sea-quark mass $m_{q}$ is corrected by its residual mass, i.e., $m_{q} \rightarrow m_{q}+m_{\text {res }}$.

\section{Results}

Using the valence quark propgator with quark mass equal to the sea-quark mass, we compute the time-correlation function of the pseudoscalar interpolator

$$
C_{\pi}(t)=\sum_{\vec{x}} \operatorname{tr}\left\{\gamma_{5}\left(D_{c}+m_{q}\right)_{0, x}^{-1} \gamma_{5}\left(D_{c}+m_{q}\right)_{x, 0}^{-1}\right\}
$$

where the trace runs over the Dirac and color space. Then the ensemble average $\left\langle C_{\pi}(t)\right\rangle$ of each $m_{q}$ is fitted to the formula $\left(Z /\left(2 M_{\pi} a\right)\right)\left[e^{-M_{\pi} a t}+e^{-M_{\pi} a(T-t)}\right]$ to extract the pion mass $M_{\pi} a$ and the decay constant $F_{\pi} a=\left(m_{q} a \sqrt{2 Z}\right) /\left(M_{\pi}^{2} a^{2}\right)$.

In Fig. B], we plot $M_{\pi}^{2} / m_{q}$ and $F_{\pi}$ versus $m_{q}$ respectively. Here we have made the correction for the finite volume effect using the estimate within ChPT calculated up to $\mathscr{O}\left(M_{\pi}^{4} /\left(4 \pi F_{\pi}\right)^{4}\right)$ [ए]], since our simulation is done on a finite volume lattice with $M_{\pi} L \sim 2.0$ for the lightest sea quark, and its finite volume effect cannot be neglected.

Taking into account of the correlation between $M_{\pi}^{2} / m_{q}$ and $F_{\pi}$ for the same sea-quark mass, we fit our data to the formulas of NLO ChPT [ए0]

$$
\begin{aligned}
\frac{M_{\pi}^{2}}{m_{q}} & =\frac{2 \Sigma}{F^{2}}\left[1+\left(\frac{\Sigma m_{q}}{16 \pi^{2} F^{4}}\right) \ln \left(\frac{2 \Sigma m_{q}}{F^{2} \Lambda_{3}^{2}}\right)\right], \\
F_{\pi} & =F\left[1-\left(\frac{\Sigma m_{q}}{8 \pi^{2} F^{4}}\right) \ln \left(\frac{2 \Sigma m_{q}}{F^{2} \Lambda_{4}^{2}}\right)\right],
\end{aligned}
$$

where $\Lambda_{3}$ and $\Lambda_{4}$ are related to the low energy constants $\bar{l}_{3}$ and $\bar{l}_{4}$ as follows.

$$
\bar{l}_{3}=\ln \left(\frac{\Lambda_{3}^{2}}{m_{\pi^{ \pm}}^{2}}\right), \quad \bar{l}_{4}=\ln \left(\frac{\Lambda_{4}^{2}}{m_{\pi^{ \pm}}^{2}}\right), \quad m_{\pi^{ \pm}}=0.140 \mathrm{GeV} .
$$

The strategy of our data fitting is to search for the values of the parameters $\Sigma, F, \Lambda_{3}$ and $\Lambda_{4}$ such that they minimize

$$
\chi^{2}=\sum_{i=1}^{8} V_{i}^{T} C_{i}^{-1} V_{i}, V_{i}=\left(\begin{array}{c}
\left(M_{\pi}^{2} / m_{q}\right)_{i}-\left(M_{\pi}^{2} / m_{q}\right)_{i}^{\mathrm{ChPT}} \\
\left(F_{\pi}\right)_{i}-\left(F_{\pi}\right)_{i}^{\mathrm{ChPT}}
\end{array}\right),
$$

where $C_{i}$ is the $2 \times 2$ covariance matrix for $M_{\pi}^{2} / m_{q}$ and $F_{\pi}$ with the same sea-quark mass, and the matrix elements of $C_{i}$ are estimated using the binning method followed by the jackknife.

For eight sea-quark masses corresponding to pion masses in the range $230-580 \mathrm{MeV}$, our fit gives

$$
\begin{aligned}
\Sigma & =[0.21855(75)(50) \mathrm{GeV}]^{3}, & F & =0.08339(35)(38) \mathrm{GeV} \\
\bar{l}_{3} & =4.149(35)(14), & \bar{l}_{4} & =4.582(17)(20),
\end{aligned}
$$




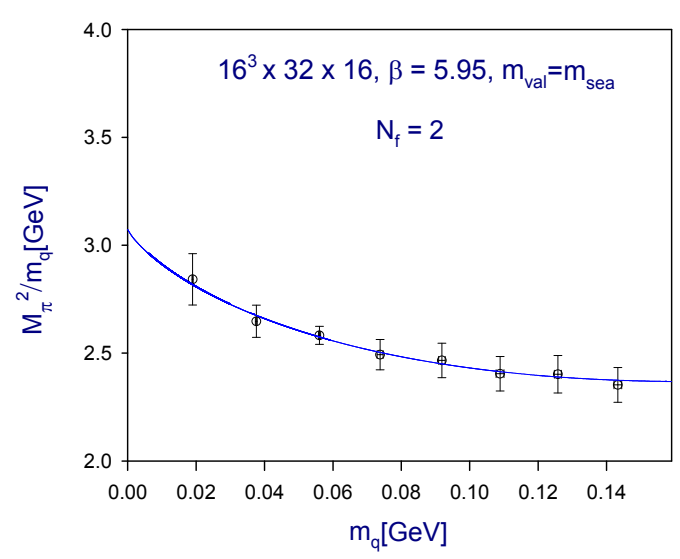

(a)

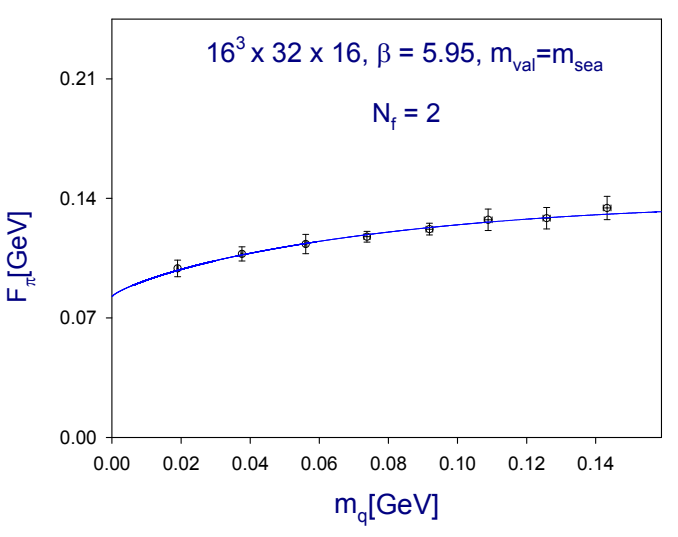

(b)

Figure 3: Physical results of 2 flavors QCD with ODWF (a) $M_{\pi}^{2} / m_{q}$, and (b) $F_{\pi}$. The solid lines are the simultaneous fits to the NLO ChPT, for eight sea-quark masses.

where the systematic errors are estimated by varying the number of data points from $8\left(M_{\pi} \leq 580\right.$ $\mathrm{MeV})$ to $5\left(M_{\pi} \leq 480 \mathrm{MeV}\right)$.

With the fitted parameters, we use the physical ratio

$$
\left(\frac{M_{\pi}}{F_{\pi}}\right)^{\text {phys }}=\frac{0.135 \mathrm{GeV}}{0.093 \mathrm{GeV}}=1.45
$$

as the input, and solve for $M_{\pi}\left(m_{q}\right) / F_{\pi}\left(m_{q}\right)=1.45$, to obtain the physical bare quark mass $m_{q}^{\text {phys }}=$ $0.00505(13) \mathrm{GeV}$. From (B.2) and (B.D), we obtain the pion decay constant and the pion mass at the physical point,

$$
\begin{aligned}
F_{\pi} & =0.090(4)(2) \mathrm{GeV} \\
M_{\pi} & =0.130(5)(3) \mathrm{GeV}
\end{aligned}
$$

Since we have used the physical ratio 1.45 as the input, in principle, we can only regard either (B.4) or (B.5) as our predicted physical result.

In order to convert the chiral condensate $\Sigma$ and the average $m_{u}$ and $m_{d}$ to those in the $\overline{\mathrm{MS}}$ scheme, we calculate the renormalization factor $Z_{s}^{\overline{\mathrm{MS}}}(2 \mathrm{GeV})$ using the non-perturbative renormalization technique through the RI/MOM scheme []], which gives $Z_{s}^{\overline{\mathrm{MS}}}(2 \mathrm{GeV})=1.244(18)(39)$. Then the values of $\Sigma$ and the average of $m_{u}$ and $m_{d}$ are transcribed to

$$
\begin{gathered}
\Sigma^{\overline{\mathrm{MS}}}(2 \mathrm{GeV})=[235(8)(4) \mathrm{MeV}]^{3}, \\
m_{u d}^{\overline{\mathrm{MS}}}(2 \mathrm{GeV})=4.06(10)(12) \mathrm{MeV}
\end{gathered}
$$

Our results of the chiral condensate (B.6) and the pion decay constant (B.4) are in good agreement with our recent results extracted from the topological susceptibility [ $[\mathbf{]}]$. Since our calculation is done at a single lattice spacing, the discretization error cannot be quantified reliably, but we do not expect much larger error because our lattice action is free from $O(a)$ discretization effects. 
To compare our results with those obtained by other lattice groups, we rely on the recent review [18]]. In general, our results of the $S U(2)$ low-energy constants, the chiral condensate and the average up and down quark mass are compatible with those obtained by other lattice groups.

\section{Concluding remark}

Our results of the mass and the decay constant of the pseudoscalar meson are in good agreement with the sea-quark mass dependence predicted by the next-to-leading order (NLO) ChPT, and provide a determination of the low-energy constants $\bar{l}_{3}$ and $\bar{l}_{4}$, the pion decay constant, the chiral condensate, and the average up and down quark mass. Together with our recent result of the topological susceptibility [ $[$ ] $]$, these suggest that the nonperturbative chiral dynamics of the sea quarks are well under control in our HMC simulations. Moreover, this also implies that it is feasible to perform large-scale simulations of unquenched lattice QCD with ODWF, which not only preserve the chiral symmetry to a high precision, but also sample all topological sectors ergodically. This provides a new strategy to tackle QCD nonperturbatively from the first principles.

This work is supported in part by the National Science Council (Nos. NSC99-2112-M-002012-MY3, NSC99-2112-M-001-014-MY3) and NTU-CQSE (No. 10R80914-4). We also thank NCHC and NTU-CC for providing facilities to perform part of our calculations.

\section{References}

[1] D. B. Kaplan, Phys. Lett. B 288, 342 (1992); Nucl. Phys. Proc. Suppl. 30, 597 (1993).

[2] H. Neuberger, Phys. Lett. B 417, 141 (1998); R. Narayanan and H. Neuberger, Nucl. Phys. B 443, 305 (1995).

[3] T. W. Chiu, Phys. Rev. Lett. 90, 071601 (2003); Nucl. Phys. Proc. Suppl. 129, 135 (2004)

[4] T. W. Chiu et al. [TWQCD Collaboration], PoS LAT2009, 034 (2009).

[5] N. I. Akhiezer, "Theory of approximation", Dover, New York, 1992.

[6] T. W. Chiu, T. H. Hsieh, C. H. Huang and T. R. Huang, Phys. Rev. D 66, 114502 (2002).

[7] T. W. Chiu, T. H. Hsieh and Y. Y. Mao [TWQCD Collaboration], Phys. Lett. B 702, 131 (2011).

[8] Y. Y. Mao and T. W. Chiu [TWQCD Collaboration], Phys. Rev. D 80, 034502 (2009).

[9] T. W. Chiu, T. H. Hsieh and Y. Y. Mao [TWQCD Collaboration], arXiv:1109.3675 [hep-lat].

[10] J. Gasser and H. Leutwyler, Nucl. Phys. B 250, 465 (1985).

[11] T. Takaishi and P. de Forcrand, Phys. Rev. E 73, 036706 (2006).

[12] J. C. Sexton and D. H. Weingarten, Nucl. Phys. B 380, 665 (1992).

[13] T. W. Chiu et al. [TWQCD Collaboration], PoS LATTICE2010, 030 (2010), and references therein.

[14] M. Hasenbusch, Phys. Lett. B 519, 177 (2001).

[15] T. W. Chiu, arXiv:hep-lat/0303008

[16] G. Colangelo, S. Durr and C. Haefeli, Nucl. Phys. B 721, 136 (2005).

[17] G. Martinelli, C. Pittori, C. T. Sachrajda, M. Testa and A. Vladikas, Nucl. Phys. B 445, 81 (1995).

[18] G. Colangelo, S. Durr, A. Juttner, L. Lellouch, H. Leutwyler, V. Lubicz, S. Necco, C. T. Sachrajda et al., Eur. Phys. J. C71, 1695 (2011). [arXiv:1011.4408 [hep-lat]], and references therein. 